\title{
Simple ELISA using Polyclonal Antibody and Lectin for Determination of Soluble Chitosan - Like Polysaccharide in Bacterial Culture
}

Research Article

Takeo $\mathrm{M}^{1 *}$, Taguchi $\mathrm{S}^{1}$, Odagaki $\mathrm{S}^{1}$, Baranwal $\mathrm{P}^{1}$, Kimura $\mathrm{K}^{2}$, Negoro $\mathrm{S}^{1}$

${ }^{1}$ Department of Applied Chemistry, Graduate School of Engineering, University of Hyogo, Shosha, Himeji, Hyogo, Japan.

${ }^{2}$ Hyogo Analysis Center Co., Ltd., Seimondori, Hirohata, Himeji, Hyogo, Japan.

Abstract

We have developed a simple enzyme-linked immunosorbent assay (ELISA) for the direct determination of chitosan-like polysaccharide (CP) produced in the cultures of Citrobacter strains. CP in sample solutions was reacted with anti-CP rabbit polyclonal antibody immobilized in a 96-well microtiter plate and sandwiched with horseradish peroxidase-conjugated lectin, followed by color development by the enzyme activities. Using standard chitosan solutions, a good standard curve $\left(\mathrm{R}^{2}=0.977\right)$ was established between 0 and $250 \mu \mathrm{g} \mathrm{l}^{-1}$ with the average coefficient of variation of $7.0 \%$, and CP in the diluted culture supernatants could be directly determined. This method can apply for the direct determination of other polysaccharides in solution by selecting an inexpensive custom-made polyclonal antibody and an appropriate lectin, both of which may be commercially available.

Keywords: ELISA; Chitosan; Biopolymer; Polysaccharides; Lectin; Citrobacter.

\section{Introduction}

Several microbial polysaccharides such as xanthan, levan, and curdlan have been practically produced in industrial scales and used for the production of foods, medicines, and cosmetics [13]. To reduce the production costs, researchers have made their best efforts to improve the productivity, which has been evaluated as the wet weight or the dry weight of the target polysaccharide produced per liter after the recovery as solid from the culture supernatants $[2,3]$. However, the recovered materials may still contain large amounts of impurities. In addition, their biological and physiological activities do not always respond to the weight measured. Therefore, it is very useful if the major structures of polysaccharides or structures essential for their activities can be directly and easily evaluated in culture supernatant or solution.

We are studying a chitosan-like polysaccharide (CP) with the average molecular weight of $>1.66 \mathrm{MDa}$ produced from acetate by Citrobacter strains, which has strong flocculating activity for suspended solids in water, and thus, can be used as a flocculant [4-6]. This biopolymer can be recovered as solid by ethanol precipita- tion and re-solubilized with acidic solutions for the use. Analyses of the dry weight and the sugar content of the recovered solid may provide good information on the amount of the CP in the original culture, but these analyses are not specific to the target polysaccharide as described above. In addition, they require long analytical time, laborious operational steps, and considerable amounts of the culture. Therefore, in the production of other microbial polysaccharides, more rapid and selective assays based on specific protein binding have been employed to quantify them [7-11]. Enzyme-linked immunosorbent assay (ELISA) is one of such methods $[12,13]$ and can detect a biomolecule of interest sensitively even in the presence of impurities, because it is based on specific antigen-antibody reaction. ELISA is well automated with the use of 96-well microtiter plates, a plate washer, and a plate reader, and thus, it has been used in many laboratories and facilities as a conventional analytical method [12]. Only the bottle neck to use ELISA is preparation of specific antibodies, which is a laborious work and very costly. Instead of antibody, lectins, which are glycan-specific binding proteins without enzyme activity [14], have also been used in similar assays [called enzyme-linked lectin or lectin sorbent assay (ELLA)] for the detection of poly-

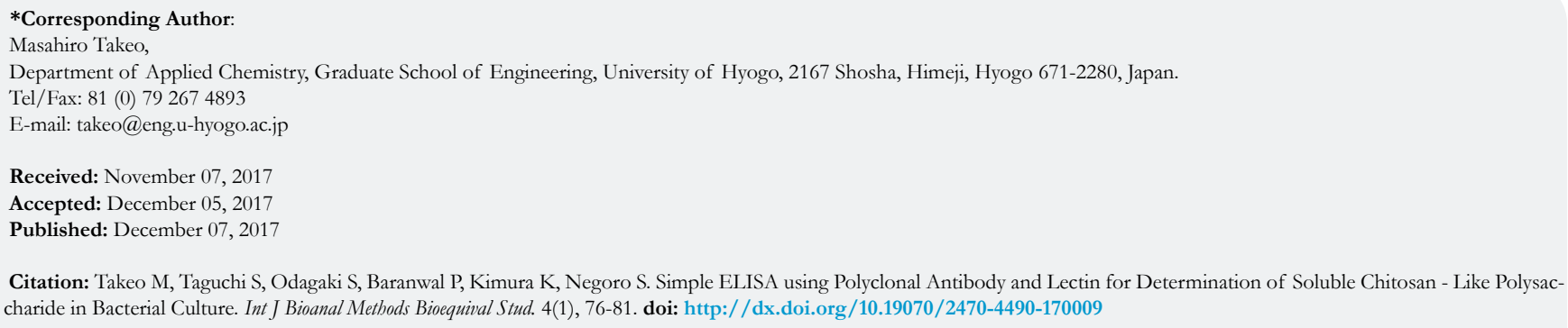

Copyright: Takeo $\mathbf{M}^{\circ}$ 2017. This is an open-access article distributed under the terms of the Creative Commons Attribution License, which permits unrestricted use, distribution and reproduction in any medium, provided the original author and source are credited. 
saccharides and glycoproteins [15-20]. Lectins can be obtained from many biosources such as bacteria, plants, and animals [14], and many lectins are commercially available. One of the representative lectins, wheat germ agglutinin (WGA), is known to preferentially recognize $N$-acetyl- $D$-glucosamine in polysaccharides $[14,21,22]$, the major constituent sugar of chitin/chitosan and the CP. Therefore, WGA has been used for the detection of chitin/chitosan in fungal and yeast cells and environmental samples [23-25].

In this study, to construct a rapid, selective, and direct determination method for the CP in solution, we employed a simple sandwich ELISA, in which a custom-made polyclonal antibody and a horseradish peroxidase (HRP)-conjugated WGA were used as a capture antibody and instead of a detection antibody, respectively. The established method recognized not only the CP but also authentic chitin/chitosan from shrimp shells even in a low concentration range. The CP amounts in samples could be determined as chitosan-mg unit using the standard curve of authentic chitosan solutions.

\section{Materials and Methods}

\section{Preparation and Purification of CP and anti-CP Rabbit IgG}

Citrobacter freundii IFO13545 (equal to NBRC13545) was cultivated at $30^{\circ} \mathrm{C}$ and $120 \mathrm{rpm}$ on a rotary shaker for $2 \mathrm{~d}$ in acetate

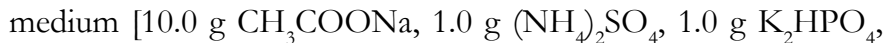
$0.05 \mathrm{~g} \mathrm{NaCl}_{0} 0.2 \mathrm{~g} \mathrm{MgSO}_{4} \cdot 7 \mathrm{H}_{2} \mathrm{O}, 0.05 \mathrm{~g} \mathrm{CaCl}_{2}, 0.01 \mathrm{~g} \mathrm{FeCl}_{3}$, and $0.1 \mathrm{~g}$ yeast extract in $1 \mathrm{l}, \mathrm{pH}$ 7.2] [4]. The culture supernatant was prepared by centrifugation $\left(8,000 \mathrm{xg}, 4^{\circ} \mathrm{C}, 10 \mathrm{~min}\right)$. Two volumes of ethanol was added to the supernatant and the mixture was kept overnight at $-20^{\circ} \mathrm{C}$ to precipitate crude $\mathrm{CP}$, which was recovered by centrifugation $\left(12,000 \mathrm{x} \mathrm{g}, 4^{\circ} \mathrm{C}, 20 \mathrm{~min}\right)$. The pellet was solubilized with a small amount of $\mathrm{HCl}$ and then dialyzed against large amounts of water in a refrigerator $\left(\right.$ at $\left.4^{\circ} \mathrm{C}\right)$. This process was repeated twice to purify CP. Finally, $7 \mathrm{mg}$ (dry weight) of CP was obtained from $100 \mathrm{ml}$ of the culture supernatant. An antiserum against $\mathrm{CP}$ was prepared in rabbit using the purified CP sample as an antigen by Sigma-Aldrich, Tokyo, Japan. In brief, the CP sample was solubilized with a small amount of $1 \%(\mathrm{v} / \mathrm{v})$ acetic acid, mixed with an adjuvant, and used to immunize an SPF grade rabbit (Japanese white) three times bi-weekly. After 56 days, the rabbit was bleeded out and $53 \mathrm{ml}$ of the antiserum was obtained from the blood. The anti-CP IgG was purified from $10 \mathrm{ml}$ of the antiserum using a Protein A affinity column by the same company. The final concentration of the purified anti-CP IgG sample $(1.7 \mathrm{ml})$ was $20.5 \mathrm{mg}$-protein $\mathrm{ml}^{-1}$ and the sample was stored at $-20^{\circ} \mathrm{C}$ as $50 \%$ (v/v) glycerol solution including $15 \mathrm{ppm}$ ProClin 300 (Sigma-Aldrich, St. Louis, MO, USA) prior to the use.

\section{Standard Sandwich ELISA for the Determination of CP}

In this ELISA, the exclusive solutions contained in the Protein Detector HRP Microwell Kit, Anti-Rabbit (SeraCare Life Science, Gaithersburg, MD, USA) were routinely used for convenience.

The standard ELISA (Figure 1) was conducted as follows; 100 $\mu \mathrm{l}$ of the anti-CP IgG solution $\left(10 \mu \mathrm{g} \mathrm{ml}^{-1}\right)$ diluted with Coating Solution was dispensed into each well of a 96-well microtiter plate (MaxiSorp, Thermo Fisher Science, Yokohama, Japan), and the plate was left overnight in a refrigerator for immobilization. After removing the solution, $250 \mu \mathrm{l}$ of BSA Diluent/Blocking Solution was dispensed into each well. Then, the plate was kept at $37^{\circ} \mathrm{C}$ for $20 \mathrm{~min}$ for blocking. After removing the blocking solution, $100 \mu$ l of sample solutions including CP, chitosan (Low MW, from shrimp shells, deacetylation 93.1\%, Sigma-Aldrich), D-glucosamine (GlcN) (Nacalai Tesque, Kyoto, Japan), N-acetyl-D-glucosamine (GlcNAc) (Nacalai Tesque), chitopentose (Funakoshi, Tokyo, Japan) or N, N', N", N',' N"',-pentaacetyl chitopentose (Funakoshi) was poured into each well. These sample solutions were prepared by diluting the original samples appropriately with Coating Solution. Antigen-antibody reaction was performed at $25^{\circ} \mathrm{C}$ for $1 \mathrm{~h}$, and then, the solution was removed. Each well was washed three times with $300 \mu$ l of Wash Solution. Then, a lectinHRP conjugate solution was prepared by diluting WGA-HRP (J-Oil Mills, Tokyo, Japan) with BSA Diluent/Blocking Solution at $0.5 \mu \mathrm{g} \mathrm{ml}^{-1}$. The lectin-HRP conjugate solution $(100 \mu \mathrm{l})$ was added to each well and kept at $25^{\circ} \mathrm{C}$ for $30 \mathrm{~min}$ to sandwich $\mathrm{CP}$ or authentic chitosan (trapped by the anti-CP IgG immobilized

Figure 1. Schemes of ELISA Steps.

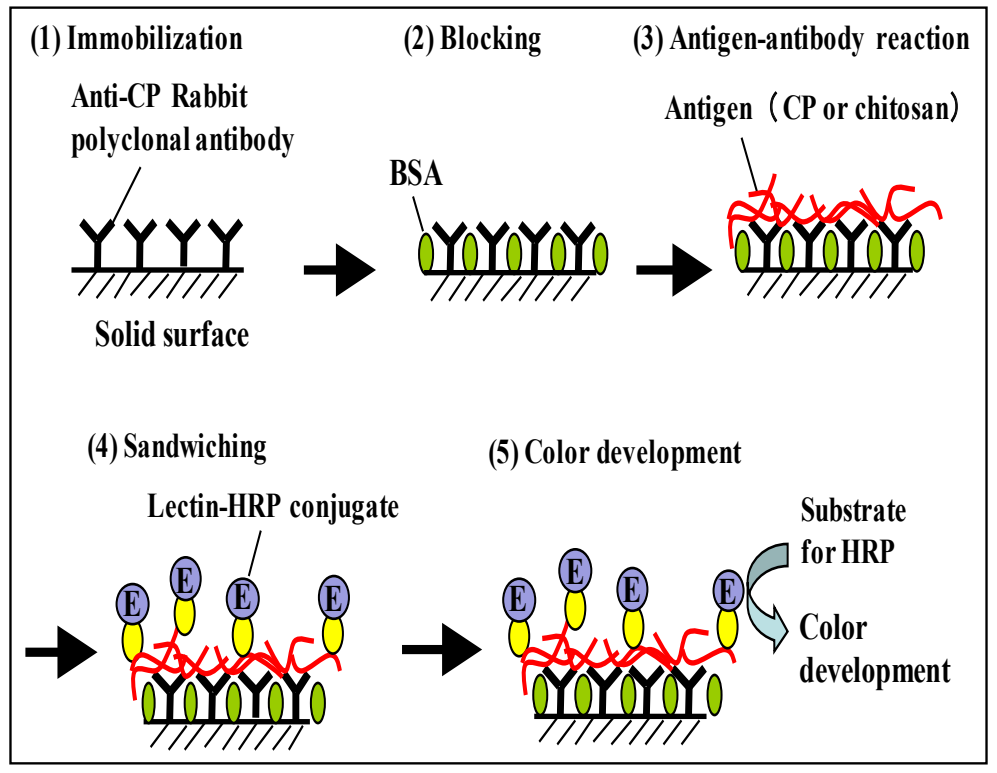


on each well) with the lectin-HRP conjugate. After removing the solution, $100 \mu \mathrm{l}$ of ABTS [2,2'-azino-bis(3-ethylbenzthiazoline6-sulfonic acid)] Enzyme Substrate Solution was introduced into each well and the solution was kept at $37^{\circ} \mathrm{C}$ for $15 \mathrm{~min}$ to develop color. Then, $100 \mu$ l of Stop Solution was added to each well and the absorbance at $405 \mathrm{~nm}$ was measured after mixing. All the measurements were carried out in triplicate.

The kit exculsive solutions can be replaced with the following solutions; Coating Solution, $10 \mathrm{mM}$ phosphate-buffered saline (PBS) (pH7.2); BSA Diluent/Blocking Solution, 1\% (w/v) BSA in $100 \mathrm{mM}$ PBS (pH7.2); Wash Solution, $100 \mathrm{mM}$ PBS (pH7.2) with $0.05 \%$ (v/v) Tween 20; ABTS Enzyme Substrate Solution, $1 \mathrm{mM}$ ABTS, $2 \%$ (v/v) $\mathrm{H}_{2} \mathrm{O}_{2}$ in $100 \mathrm{mM}$ citrate buffer (pH4.5); Stop Solution, $1 \%$ SDS (w/v) in water. Except for dispensing the samples, most processes (dispensing the reagent solutions, mixing by shaking the plate, incubation at directed temperature, aspiration, and measurement of absorbance) were automatically carried out using a Crocodile Mini-Workstation (Titertek-Berthold, Pforzheim, Germany). A stock chitosan solution was prepared at $100 \mathrm{mg} \mathrm{l}^{-1}$ by dissolving $5 \mathrm{mg}$ of the authentic chitosan (SigmaAldrich) with $50 \mathrm{ml}$ of $1 \%(\mathrm{v} / \mathrm{v})$ acetic acid. Then, standard chitosan solutions were prepared between 0 and $250 \mu \mathrm{g} \mathrm{l}^{-1}$ by diluting the stock solution with Coating Solution.

\section{Results and Discussion}

The CP of C. freundii IFO13545 produced from acetate has a very similar structure to that of chitin/chitosan $[4,26]$. Therefore, commercially available authentic chitosan from shrimp shells (Sigma-Aldrich) was used to make standard solutions for this assay. As shown in Figure 2, using the standard chitosan solutions, a good standard curve $\left(\mathrm{R}^{2}=0.977\right)$ was established between 0 and $250 \mu \mathrm{g} \mathrm{l}^{-1}$ with the average coefficient of variation of $7.0 \%$, although the polyclonal antibody (anti-CP rabbit $\mathrm{IgG}$ ) used was prepared against the CP of IFO13545 in rabbit. This proves that the CP of IFO13545 is very similar in structure to the authentic chitosan.

From the 2-d cultures of $C$. freundii IFO13545, C. freundii IFO 13545 $b f p C$ (a CP-deficient mutant of IFO13545), and Escherichia coli JM109 (Takara Bio, Kyoto, Japan), each culture supernatant was prepared by centrifugation $\left(12,000 \times \mathrm{g}, 4^{\circ} \mathrm{C}, 20 \mathrm{~min}\right)$, and then, the 10 -fold dilution series of the culture supernatants were prepared with Coating Solution. The diluted samples were subjected to the established ELISA. A medium control (without the inoculation of the bacterial cells) was also prepared and measured in the same way. As shown in Figure 3, the absorbance of the diluted samples of the $C$. freundii IFO13545 culture supernatant started to decline at the $10^{3}$-fold dilution, sharply decreased, and reached an almost basal level at the $10^{4}$-fold dilution (Run1). To obtain the detailed data between the $10^{3}$-fold and $10^{4}$-fold dilutions, the culture supernatant of IFO13545 was again diluted at narrow intervals and the diluted samples were measured as the second trial. As a result, the obtained data points formed a smooth declining curve in the dilution range (Figure 3, Run2). As the absorbance at $405 \mathrm{~nm}$ of the 3000 -fold diluted sample was 2.096 (Figure 3), the CP concentration was determined $155 \mu \mathrm{g}$ chitosan $\mathrm{l}^{-1}$ from the standard curve of authentic chitosan (Figure 2), being equal to $464 \mathrm{mg}$-chitosan $\mathrm{l}^{-1}$ in the original culture supernatant. These results demonstrate that it is possible to quantify $\mathrm{CP}$ in culture supernatant relatively as chitosan-mg unit. This is very similar to the determination of protein concentrations in solution using bovine serum albumin as a standard. In contrast, the absorbance of the medium control was consistent at a basal level regardless of the dilutions (Figure 3), indicating that the medium components did not affect the CP measurement significantly. In addition, the diluted samples of the $C$. freundii IFO13545 $\Delta b f p C$ (a CP-deficient mutant) and E. coli JM109 culture supernatants were also at the basal level, although the non-diluted and the 10 -fold diluted samples showed somewhat higher values than the basal level. Even this background absorbance could be completely ignored if the samples to be measured are more than $10^{2}$-fold diluted with Coating Solution.

The polyclonal antibody used might contain a small amount of IgG against impurities such as proteins, nucleotides, and other polysaccharides, because the purified CP sample used for immunization still might contain a trace amount of the impurities. Therefore, in this ELISA, these impurities also might be caught on the polyclonal antibody immobilized on the wells, nevertheless, the background was low and consistent, compared to the detection level of the CP (Figure 3). This is probably due to double selections by the polyclonal antibody and the lectin-HPR conjugate.

Figure 2. Standard Curve using Authentic Chitosan from Shrimp Shell (Low MW, Sigma).

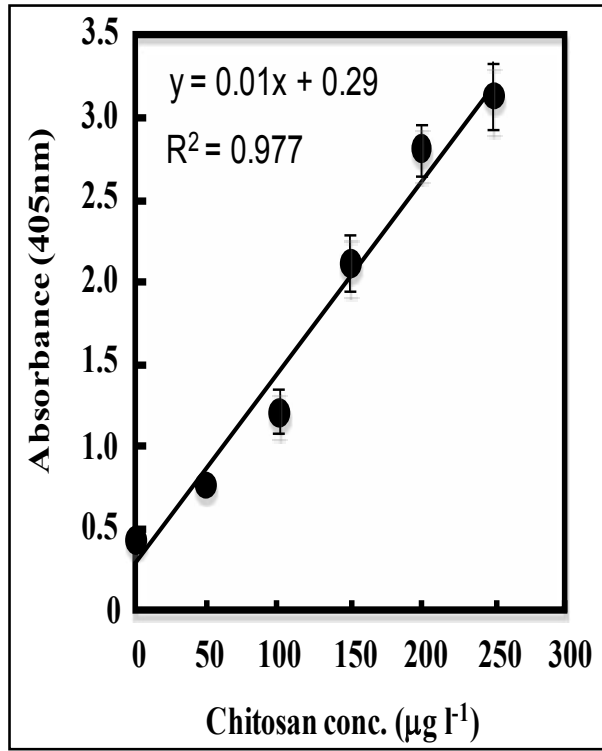

Takeo M, Taguchi S, Odagaki S, Baranwal P, Kimura K, Negoro S. Simple ELISA using Polyclonal Antibody and Lectin for Determination of Soluble Chitosan - Like Polysaccharide 
Figure 3. Measurement of CP in the Culture Supernatants of $C$. freundii IFO13545 (Run1, closed circle: Run2, open circle), C. freundii IFO13545 $\triangle b f p C$ (open triangle), Escherichia coli JM109 (closed triangle ), and medium control (closed square).

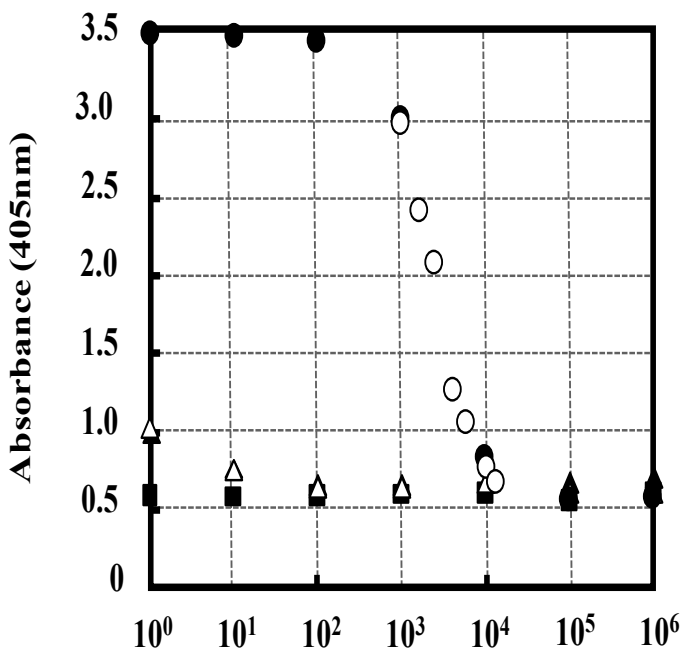

Dilution of culture supernatant

In the competitive ELISA for the determination of 2-substituted $(1,3)-\beta$ - $D$-glucans in the cultures of lactic acid bacteria, polyclonal antibody was used to detect the $\beta$-glucans [11]. This assay detected the target $\beta$-glucans sensitively (in the range of 20 to $2500 \mu \mathrm{g} \mathrm{ml}$ $\left.{ }^{1}\right)$, but competition with another $\beta$-glucan [6-substituted $(1,3)-\beta$ - $D$ glucan] was observed in the lower concentration range (10 to 250 $\left.\mu \mathrm{g} \mathrm{ml}^{-1}\right)$ [11]. Single use of polyclonal antibody in ELISA tends to cause unexpected cross reactions, which result in the inaccurate determination of the target polysaccharide. The sandwich assay employed in this study can improve this problem by the additional selection with WGA.

WGA has been frequently used to detect the biofilm components (exopolysaccharides) of bacterial strains and the cell wall components (chitin/chitosan) in fungal and yeast cells [16, 17, 19, 23, 24], because WGA recognizes GlcNAc and its consecutive oligo units in polysaccharides [14, 21, 22]. Due to this specificity, GlcNAc and the oligomers work as strong competitive inhibitors for the binding of WGA to the target polysaccharides and glycoproteins $[16,21,25,27]$. The acetate medium used for the cultivation of the CP-producing strain contains yeast extract, which may provide GlcNAc and the oligomers from disrupted yeast cell walls. In addition, in aged cultures, they may be released from the peptido glycan of dead bacterial cells. Therefore, the presence of GlcNAc and the oligomers in the culture should be considered.

To know the effect of GlcNAc and the oligomers, GlcN, GlcNAc, chitopentose $\left[(\mathrm{GlcN})_{5}\right]$, and N,N',N",N",'N"' -pentaacetyl chitopentose $\left[(\mathrm{GlcNAc})_{5}\right]$ were also measured at various concentrations by this sandwich ELISA. However, the absorbance of these samples did not increase from the basal level even at $>1 \mathrm{mg}$ $\mathrm{l}^{-1}$ (Figure 4). Therefore, these compounds are unlikely to affect the measurement of the CP by this method.

In simple ELLAs, a target polysaccharide or a glycoprotein in sample is first immobilized on microtiter plate wells, and then, detected by a lectin conjugated with an indicator enzyme such as HRP. In this case, the presence of the competitive inhibitors can affect the binding of the lectin to the target molecule significant- ly. In fact, in the detection of GlcNAc in the biofilm matrix of Staphylococcus epidermidis by ELLA, GlcNAc inhibited the detection strongly [16]. Since the present method did not detected GlcNAc and the pentamer even at high concentrations (Figure 4), they might be washed out through the first capture reaction with the polyclonal antibody and the subsequent washing process.

In some ELLAs, in turn, a lectin is first coated on microtiter plate wells to catch the target molecule in sample, and then, specific antibody against the target molecule is used to detect it [28]. This case would be also affected by the inhibitors, because the inhibitors in sample compete with the target polysaccharide for the binding to the coated lectin in the first capture reaction. Accordingly, we think that our sandwich style consisting of the first capture reaction with polyclonal antibody and the second sandwiching reaction with lectin is most suitable to lower the effect of the low molecular competitive inhibitors.

In many simple ELISAs and ELLAs, a target molecule in sample is first coated on microtiter plate wells. This type needs enough time to immobilize the target molecule on solid surface. For example, in the ELLA for the detection of the extracellular polysaccharides (EPSs) of Streptococcus mutans, Decker et al. employed a 20-h incubation time to immobilize the EPSs to the microtiter plate wells [18]. Werning et al. also employed a 16-h incubation time to immobilize the $\beta$-glucans of lactic acid bacteria in the ELISA [11]. Such long times are not suitable for the monitoring of microbial polysaccharides in growing cultures. The present method can complete the measurement in approximately 2 $\mathrm{h}$, if the polyclonal antibody is immobilized to the microtiter plate wells prior to the test day.

Similar sandwich ELISAs containing lectin have been widely used for the detection of biomarkers for cancers and glycoproteins in medical samples [19, 20]. However, no reports on similar ELISA (antibody/lectin sandwich method) could be found for the direct determination of microbial polysaccharides in solution. The present method was established within 6 months including the antibody preparation period and at a low cost by using an inexpensive 
Figuer 4. Measurement of $D$-glucosamine (GlcN) and $N$-acetyl- $D$-glucosamine (GlcNAc) (A), and their pentamers (B).

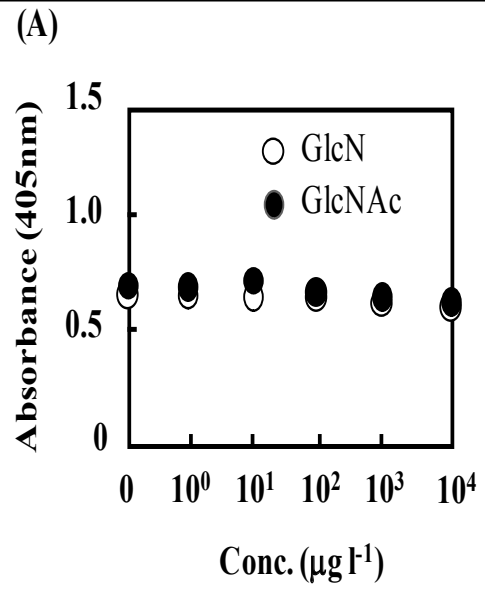

(B)

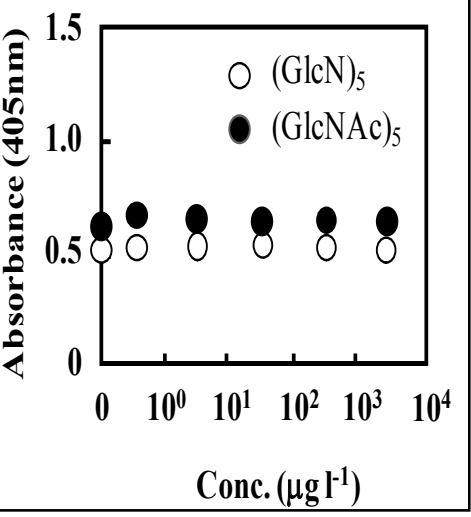

custom-made polyclonal antibody and a commercially available WGA. The result shown here is only a case study, but this methodology can apply for the rapid establishment of the direct determination method for polysaccharides in solution, if an appropriate lectin specific to the target polysaccharide can be obtained.

\section{Acknowledgements}

This work was supported in part by the Regional Innovation Strategy Support Program (2012-2017), Ministry of Education, Culture, Sports, Science and Technology, Japan, and a Grant-in Aid for Scientific Research (C) from Japan Society for the Promotion of Science (JSPS) (2015-2017), and the JSPS bilateral collaboration program with the Curtin Univ. (Perth, Australia) research team (2016-2017).

\section{References}

[1]. Freitas F, Alves VD, Reis MA. Advances in bacterial exopolysaccharides: from production to biotechnological applications. Trends Biotechnol. 2011 Aug;29(8):388-98. PubMed PMID: 21561675.

[2]. Donot F, Fontana A, Baccou JC, Schorr-Galindo S. Microbial polysaccharides: main examples of synthesis, excretion, genetics and extraction. Carbohydr Polym. 2012 Jan;87(2):951-62.

[3]. Öner ET. Microbial production of extracellular polysaccharides from biomass. In: Fang Z, Editor. "Pretreatment techniques for biofuels and biorefineries”. Springer NY. 2013 Jan;35-55.

[4]. Kimura K, Inoue T, Kato D, Negoro S, Ike M, Takeo M. Distribution of chitin/chitosan like bioflocculant producing potential in the genus Citrobacter. Appl Microbiol Biotechnol. 2013 Nov;97(21):9569-77. PubMed PMID: 23321910.

[5]. Renault F, Sancey B, Badot P-M, Crini G. Chitosan for coagulation/flocculation processes-an eco-friendly approach. European Polymer Journal. 2009 May; $45(5): 1337-48$.

[6]. Fujita M, Ike M, Jang JH, Kim SM, Hirao T. Bioflocculation production from lower-molecular fatty acids as a novel strategy for utilization of sludge digestion liquor. Water Sci Technol. 2001;44(10):237-43. PubMed PMID: 11794660 .

[7]. Golman S, Shabtai Y, Rubinovitz C, Rosenberg E, Gutnick DL. Emulsan in Acinetobacter calcoaceticus RAG-1: distribution of cell-free and cell-associated cross-reacting material. Appl Environ Microbiol. 1982 Jul;44(1):165-70. PubMed PMID: 16346052

[8]. Haaheim LR, Kleppe G, Sutherland IW . Monoclonal antibody reacting with the exopolysaccharide xanthan from Xanthomonas campestris. J Gen Microbiol. 1989 Mar;135(3):605-12. PubMed PMID: 2621443.

[9]. Davies DG, Chakrabarty AM, Geesey GG .Exopolysaccharide production in biofilm:substream activation of alginate gene expression by Pseudomonas aeruginosa. Appl Environ Microbiol. 1993 Apr; 59(4): 1181-6. PubMed PMID: 202258.

[10]. Izawa N, Hanamizu T, Iizuka R, Sone T, Mizukoshi H, Kimura K, Chiba $K$.
Streptococcus thermophiles produces exopolysaccharides including hyaluronic acid. J Biosci Bioeng. 2009 Feb;107(2):119-23. PubMed PMID: 19217548.

[11]. Werning ML, Perez-Ramos A, Fermandez de Palencia P, Mohedano ML, Duenas MT, Prieto A, López P. A specific immunological method to detect and quantify bacterial 2 -substituted $(1,3)-\beta$ - $D$-glucan. Carbohydr Polym. 2014 Nov 26;113:39-45. PubMed PMID: 25256456.

[12]. Lequin RM .Enzyme immunoassay (EIA)/enzyme-linked immunosorbent assay (ELISA). Clin Chem. 2005 Dec;51(12):2415-8. PubMed PMID: 16179424.

[13]. Michael J. Introduction to antigen and antibody assays. Top Companion Anim Med. 2015 Dec;30(4):128-31. PubMed PMID: 27154595.

[14]. Lis H, Sharon N. Lectins: carbohydrate-specific proteins that mediate cellular recognition. Chem Rev. 1998 Apr 2;98(2):637-74. PubMed PMID: 11848911.

[15]. Haines J, Patel PD. Antibody- and lectin-based assays for the rapid analysis of food grade gums and thickeners. Trends Food Sci Technol.1997 Dec;8(12):395-400.

[16]. Thomas VL, Sanford BA, Moreno R, Ramsay MA. Enzyme-linked lectin sorbent assay measures $\mathrm{N}$-acetyl-D-glucosamine in matrix of biofilm produced by Staphylococcus epidermidis. Current Microbiology.1997 Oct; 35(4):249-54.

[17]. Leriche V, Sibille P, Carpentier B. Use of an enzyme-linked lectinsorbent assay to monitor the shift in polysaccharide composition in bacterial biofilms. Appl Environ Microbiol. 2000 May;66(5):1851-6. PubMed PMID: 10788349.

[18]. Decker E-M, Dietrich I, Klein C, von Ohle C. Dynamic production of soluble extracellular polysaccharides by Streptococcus mutans. Int J Dent. 2011;2011:435830. PubMed PMID: 22046185.

[19]. Tang H, Hsueh P, Kletter D, Bern M, Haab B. The detection and discovery of glycan motifs in biological samples using lectins and antibodies: new methods and opportunities Adv Cancer Res. 2015;126:167-202. PubMed PMID: 25727148.

[20]. Miyoshi E, Kamada Y. Application of glycoscience to the early detection of pancreatic cancer. Cancer Sci. 2016 Oct; 107(10): 1357-62. PubMed PMID: 27418030

[21]. Allen AK, Neuberger A, Sharon N. The purification, composition and specificity of wheat germ agglutinin. Biochem J. 1973 Jan;131(1):155-62. PubMed PMID: 4737292

[22]. Peters BP, Ebisu S, Goldstein IJ, Flashner M. Interaction of wheat germ agglutinin with sialic acid. Biochemistry. 1979 Nov 27;18(24):5505-11. PubMed PMID: 92996.

[23]. Tronchin G, Poulain D, Herbaut J, and Biguet J. Localization of chitin in the cell wall of Candida albicans by means of wheat germ agglutinin. Fluorescence and ultrastructural studies. Eur J Cell Biol. 1981 Dec;26(1):121-8. PubMed PMID: 7035173.

[24]. Gueddari NEEl, Rauchhaus U, Moerschbacher BM, Deising HB. Developmentally regulated conversion of surface-exposed chitin to chitosan in cell walls of plant pathogenic fungi. New Phythologist 2002 Oct;156(1):103-12.

[25]. Montgomery MT, Welschmeyer NA, Kirchman DL. A simple assay for chitin: application to sediment trap samples from the subarctic pacific. Mar Ecol Prog Ser. 1990 July;64:301-8.

[26]. Fujita M, Ike M, Tachibana S, Kitada G, Kim SM, Inoue Z. Characterization of a bioflocculant produced by Citrobacter sp. TKF04 from acetic and propionic acids. J Biosci Bioeng. 2000; 89(1):40-6. PubMed PMID: 16232696. 
[27]. Afrough B, Dwek MV, Greenwell P. Identification and elimination of falsepositives in an ELISA-based system for qualitative assessment of glycoconjugate binding using a selection of plant lectins. Biotechniques. 2007 Oct;43(4):458, 460, 462. PubMed PMID: 18019336.

[28]. Peter N, Makin CA, Ching CK, EcclestonD, Taylar OM, Milton JD, et al.
A new enzyme-linked lectin/mucin antibody sandwich assay (CAM 17.1/ WGA) assessed in combination with CA 19-9 and peanut lectin binding assay for the diagnosis of pancreatic cancer. Cancer. 1992 Sep 1;70(5):1062-8. PubMed PMID:1515982. 